Proc. Indian Acad. Sci. (Earth Planet. Sci.), Vol. 92, Number 1, March 1983, pp. 15-30.

- Printed in India.

\title{
Analytical representation of spatial and temporal variations of the geomagnetic field in the Indian region
}

\author{
B R ARORA, V H BADSHAH, B P SINGH, M G ARUR*, \\ P S BAINS* and JEEVAN LAL* \\ Indian Institute of Geomagnetism, Colaba, Bombay 400 005, India \\ * Geodetic \& Research Branch, Survey of India, Dehradun, India
}

MS received 5 June 1982; revised 30 September 1982

\begin{abstract}
The magnetic measurements of declination $(D)$, horizontal $(H)$ and vertical $(Z)$ components of earth's magnetic field, collected from ground surveys between 1962 and 1966 , are used to develop an analytical model of geomagnetic field variations over Indian region for the epoch 1965. In order to reflect spatial features with wavelengths of approximately $1000 \mathrm{~km}$, sixth degree polynomial as a function of differential latitude and longitude is calculated by the method of least squares. The root mean square fit of the model to the input data is better than that accounted by the International Geomagnetic Reference Field for 1965.0. Isomagnetic charts drawn for $D, H, Z$ and total force $(F)$ reflect more details than that shown on world magnetic charts. Further, the values of the field at common repeat stations recorded between 1962 and 1974, after eliminating the field values for the epoch 1965.0, are used to get the secular variation as well as its spatial dependence again by means of polynomial which now includes coefficients which are functions of time and of geographical locations. The accuracy of coefficients is tested against the behaviour of secular variation at permanent magnetic observatories. The merits and limitations of the model are discussed.
\end{abstract}

Keywords. Geomagnetic reference field; isomagnetic charts; secular variation; polynomial fit; analytical representation; spatial variation; temporal variation.

\section{Introduction}

The application of geomagnetic field measurements in delineating crustal magnetic anomalies, which are of longer wavelengths than those investigated by exploration geophysicists, is being increasingly recognised (for example see Hinze 1979; Blakely and Cande 1979). This has resulted from significant developments in the modes of data acquisition as well as due to the development of new analytical interpretation techniques. The true utilization of geomagnetic measurements for geological purposes is achieved when a normal or regional field corresponding to the main field of the earth and regional features is identified and eliminated from measurements. The definition and basis of choosing normal field has to be complimentary with the wavelengths of anomalies being sought so that fields from regional features and anomalous zones of interest do not interfere with each other.

The magnetic charts obtained through the spherical harmonic analysis of global data exhibit smooth spatial variation of field with wavelengths attributable to sources in the core. For example, spherical harmonic analysis of degree and order 12, commonly employed, depicts details of the field having spatial scales greater than $3300 \mathrm{~km}$ (the wavelength of the twelfth harmonic), comparable to the dimension of many of the subcontinents. The treatment of spherical harmonic analysis is less suitable for the 
compilation of regional charts because of the high degree and order required to reveal wavelengths which such regional charts are desired to reveal. Prior to 1965 , most regional charts were contoured by hand directly from the observed data without first fitting the data to a selected mathematical function. The process is not only subjective to human bias but also provides data in a form less adaptable to direct quantitative treatment. In the past decade, some attempts have been made to formulate regional charts analytically, wherein the field values are represented by power series in longitude and latitude (Nagata 1971). Occasionally the term corresponding to time is also included to model the secular variation of the field (Dawson and Newitt 1977; Nevanlinna 1979).

The Survey of India (soI) has been periodically conducting field magnetic surveys for graphical preparation of regional magnetic charts over Indian region primarily for navigational needs. In their endeavour to participate in the World Magnetic Surveys (wMS) during 1964-65, sol carried out an extensive survey of the country by making geomagnetic measurements at field stations in addition to a regular set of repeat stations. This survey was extended over the years 1962-66. The separation between two successive field stations ranged from 30 to $80 \mathrm{~km}$. This is in contrast to repeat stations which have interstation spacing of the order of $200-300 \mathrm{~km}$. Through the use of both field and repeat stations, isomagnetic charts were graphically compiled for the epoch of 1965.0. Subsequently, data from two other surveys corresponding to the epoch 1970.0 and 1975.0 have also become available. During these surveys, data from repeat stations only were acquired. The present study is aimed at utilizing this extensive data base in developing an analytical model representing the spatial and temporal variation of geomagnetic field in the Indian region.

\section{Data acquisition and reduction procedure}

The magnetic measurements undertaken by Survey of India consist of observations and computations of horizontal force $(H)$, vertical force $(Z)$ and declination $(D)$ at repeat and field stations using $\mathrm{QHM}, \mathrm{BMZ}$ and $\mathrm{T} 2$ theodolite respectively. The field instruments used in the survey were calibrated against the permanent geomagnetic observatory standards at Alibag. From the data, corrected for instrumental differences, the quiet-day daily variation and magnetic disturbance effects a re eliminated with the aid of data from permanent magnetic observatory close to the survey station. During the magnetic survey extending over years 1962-1966, a total of 989 field and repeat stations were covered. Of these, only 732 stations, where all the three components namely $H, Z$ and $D$ were observed, were selected for the analysis. From subsequent surveys, spreading over the years 1966-1970 and 1972-1975, data from 151 and 166 repeat stations respectively were available but all the stations covered were not common in the two surveys. The number of common repeat stations among all the three surveys was 93 . The distribution of these repeat stations as well as the field stations selected from the first survey is shown in figure 1.

\section{Analysis and results}

\subsection{Preliminary analysis}

The polynomials of geomagnetic field as a function of latitude and longitude are widely used to depict smoothlv varying geomagneric fields in limited areas. In such an 


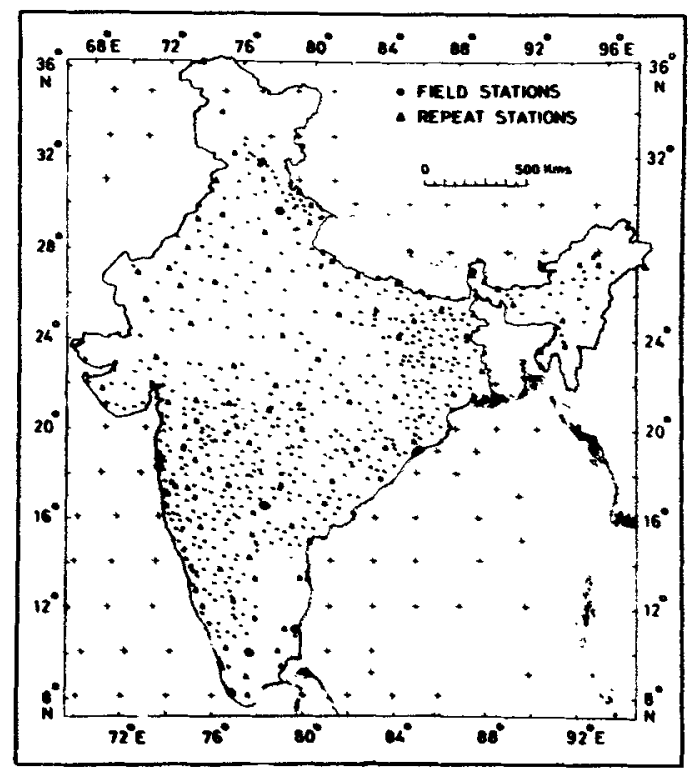

Figure 1. Distribution of magnetic field stations for the epoch of 1965.0 and repeat stations where three observations are available during 1962-75. represent permanent magnetic observations and + mark indicates locations at seas where control values evaluated from IGRF are provided.

approach, the spatial characteristics of the field to be revealed are controlled by the extent of the area and the degree of polynomial adopted. In order to produce magnetic charts for Indian region, reflecting wavelengths of about $1000 \mathrm{~km}$, it is necessary to use a polynomial fit of degree six which according to Bullard's (1967) equivalent degree method would reflect magnetic features of required wavelengths. This follows from the fact that the Indian sub-continent bounded by geographic latitudes $8^{\circ}$ to $36^{\circ} \mathrm{N}$ and longitudes $70^{\circ}$ to $96^{\circ} \mathrm{E}$ represents a mapping area of about $8.8 \times 10^{\circ} \mathrm{km}^{2}$ and a sixth degree polynomial fit with 28 coefficients to this size is nearly equivalent to a spherical harmonic fit of degree and order 40 for the world, and therefore, should reflect wavelengths close to $1000 \mathrm{~km}$. Although the knowledge of spatial characteristics of the geomagnetic field is of primary importance for geological applications, but as the geomagnetic field undergoes slow temporal variations, called secular variation, it becomes desirable that a complete description of magnetic field by analytical functions should also incorporate time coefficients in addition to spatial coefficients. The one distinct advantage of including time coefficient is that the analysis can be performed directly on the observed data by surpassing the customary laborious procedure of updating the data to certain epoch (Dawson and Newitt 1977). In view of the above discussion, the polynomial function chosen to map any component of geomagnetic field is similar to that used by Dawson and Newitt (1977) and includes sixth degree spatial terms and truncated quadratic time terms as follows:

$$
C=\sum_{i=0}^{6} \sum_{i=0}^{i} \Delta \theta^{\prime}{ }^{i} \Delta \lambda^{i} \sum_{k=0}^{2} a_{i-1, \ldots k} \Delta I^{k}
$$


In (1) $\Delta \theta=\theta-\theta_{0}, \Delta \lambda=\lambda-\lambda_{0}$ and $\Delta t=t-t_{0}$ where $\theta, \lambda$ are the latitude and longitude of observational point and $t$ is the data of observation; $\theta_{0}, \lambda_{0}$ are the geographic coordinates of the central expansion point and $t_{0}$ is a reference epoch. The $a_{i-j, j, k}$ are the coefficients to be evaluated. In the above expression, when the index $k<0$, it is taken as $k=0$. The time terms are restricted to second order for the fact that in the time domain data were available only from three surveys and, therefore, seculartrend could at best be estimated by a parabola. Using all the data base from three surveys, the set of coefficients $a$ 's in the above equation were calculated by the method of least squares separately for $H, Z$ and $D$. The geographic latitude $22^{\circ} \mathrm{N}$, longitude $80^{\circ} \mathrm{E}$ have been taken as the central point and the time deviations are reckoned with respect to 1965.0. The validity of time and spatial coefficients has been checked, respectively, by computing the temporal variation at a few magnetic observatories and by constructing the isomagnetic charts for the whole region. The temporal variation given by the expansion of polynomial at different observatories fail to reveal the features noticed on the magnetic observatory data, both in regard to form and magnitude. It is hard to ascertain the cause for the lack of compatability of modelled values with the observatory data but one probable source of ambiguity could be related with nonuniform distribution of data in time domain over different parts of the region.

The peculiar feature seen on isomagnetic charts was that the smoothly varying geomagnetic field over the continent was found to converge immediately off the boundaries of the Indian continent. Essentially such an abrupt behaviour is the artifact of the analysis approach, as no such behaviour is noticed on the world magnetic charts compiled from IGRF coefficients for 1965.0 (Fabiano and Peddie 1969). This may be attributable to the absence of observations on the adjacent seas. This preliminary exercise clearly revea is that with presently available data base, it is hard to analytically represent the magnetic field variation both in space and time by a single polynomial expression of the type described in equation (1). However, the attempt of modelling the data of first magnetic survey, which had a fairly close net of observations, after reducing them to a common epoch of 1965.0 , with recourse to obseratory data, was rewarding. After eliminating the geomagnetic field values for the epoch of 1965.0 from observations at common repeat stations of three surveys, the residual values are used to get time variation as well as its spatial dependence. The following sections describe the results of the above two approaches.

\subsection{Analytical representation of spatial variations}

The magnetic measurements, collected as part of wMS from 732 field and repeat stations obtained over a time span of 1962-1966 were reduced to the epoch of 1965.0 by applying a correction corresponding to the change in field values between the actual period of observation and epoch. Correction factors were calculated from the nearest permanent magnetic observatory with an underlying assumption that secular variation at field and reference stations is the same. These epoch reduced data are employed for mapping the spatial characteristic of the geomagnetic field variation. For the consideration already mentioned in the earlier section, sixth degree polynomial with the following form is adopted

$$
C=\sum_{i=0}^{6} \sum_{j=0}^{i} a_{i-j, j} \Delta \theta^{i-j} \cdot \Delta \lambda^{j}
$$


here all the notations have the same meaning as in (1).

In accord with the requirement of electromagnetic theory, Chapman(1942) worked out the relations between various elements and suggested that the quality of isomagnetic charts derived from measurements at discrete points would improve if certain inter-relations are satisfied in the data analysis. One such condition, under the assumption that the vertical component of $\operatorname{Curl} F=0$, relates the surface variation of $X$ (North) and $Y$ (East) elements as

$$
\frac{\delta X}{\delta \lambda}=\frac{\delta(Y \cos \theta)}{\delta \theta}=0
$$

The power series expression for $X$ and $Y \cos \theta \operatorname{can}$ be written in its expanded form from (2) as

$$
\begin{gathered}
X=a_{0}+a_{1} \Delta \theta+a_{2} \Delta \lambda+a_{3} \Delta \theta^{2}+a_{4} \Delta \theta \cdot \Delta \lambda+a_{5} \Delta \lambda^{2}+\ldots \\
+a_{27} \Delta \lambda^{6} \\
Y \cos \theta=b_{0}+b_{1} \Delta \theta+b_{2} \Delta \lambda+b_{3} \Delta \theta^{2}+b_{4} \Delta \theta \cdot \Delta \lambda+b_{5} \Delta \lambda^{2} \\
\ldots+b_{27} \Delta \lambda^{6}
\end{gathered}
$$

Using (3) as a necessary condition for consistency, a relationship between the coefficients of (4) and (5) is obtained. These are listed in table 1. These relations were applied to equation (4) and both equations (4) and (5) were expanded to include all the coefficients as follows:

$$
\begin{aligned}
& X=a_{0}(1)+b_{0}(0)+a_{1} \Delta \theta+b_{1} \Delta \lambda+b_{2}(0)+\cdots \\
& Y \cos \theta=a_{0}(0)+b_{0}(1)+a_{1}(0)+b_{1} \Delta \theta+b_{2} \Delta \lambda+\cdots
\end{aligned}
$$

The combined set of coefficients in (6) and (7) for the $X$ and $Y \cos \theta$ system is simultaneously determined by the least square solution. The coefficients were finally reduced to be compatible with (4) and (5). These are given in table 2 . The coefficients for $Z$, determined separately, are also given in table 2 . A preliminary exercise carried out in $\S 2$ has revealed that due to the lack of data in the neighbourhood of Indian continent, modelled variation showed a sharp convergence off the continental boundaries. In order to provide some control on the nature of the distribution of the field on adjacent seas, certain control values evaluated from IGRF (Fabino and Peddie 1969) were included at 76 points, prior to the computation of coefficients. The location of these points is shown in figure I as + mark. The root mean square (RMS) values derived from the difference between the observed values and the calculated values are given in table 3.

Using the coefficients corresponding to $X, Y \cos \theta$ and $Z$, the magnetic field values for $D, H, Z$ and $F$ were computed for the geographical network of $1^{\circ} \times 1^{\circ}$ grid points and isomagnetic charts drawn. Unlike the results in the previous section, the isomag- 
Table 1. Coefficient interrelation for $X$ and $Y \cos \theta$ system.

$\begin{array}{lll}a_{2}=b_{1} & a_{12}=3 b_{11} / 2 & a_{20}=b_{19} / 5 \\ a_{4}=2 b_{3} & a_{13}=2 b_{12} / 3 & a_{22}=6 b_{21} \\ a_{5}=b_{4} / 2 & a_{14}=b_{13} / 4 & a_{23}=5 b_{22} / 2 \\ a_{7}=3 b_{6} & a_{16}=5 b_{15} & a_{24}=4 b_{23} / 3 \\ a_{8}=b_{7} & a_{17}=2 b_{16} & a_{25}=3 b_{24} / 4 \\ a_{9}=b_{8} / 3 & a_{18}=b_{17} & a_{26}=2 b_{25} / 5 \\ a_{11}=4 b_{10} & a_{19}=b_{18} / 2 & a_{27}=b_{26} / 6\end{array}$

Table 2. Polynomial coefficients describing spatial variation of geomagnetic field. Central expansion point $\left(\theta, \lambda_{0}\right)$ is taken at $22^{\circ} \mathrm{N}$ and $80^{\circ} \mathrm{E}$. In variable column DLAT $=\Delta \theta$; DLON $=\Delta \lambda$; DMLT $=\Delta \mathrm{T}$. A number associated with these indicates the power of variable (DLAT $\left.2=\Delta \theta^{2}\right)$

\begin{tabular}{|c|c|c|c|c|}
\hline Variable & $\boldsymbol{X}$ & $Y \cos$ & $Z$ & Variable \\
\hline 1 & $+.38629 E+05$ & $-.78887 E+03$ & $.22125 E+05$ & $\mathbf{J}$ \\
\hline DLAT & $-.38622 E+03$ & $.10706 \mathrm{E}+03$ & $.16145 E+04$ & DLAT \\
\hline DLON & $.10706 \mathrm{E}+03$ & $-.16999 E+02$ & $-.22551 E+02$ & DLON \\
\hline DLAT2 & $-.16894 E+02$ & $.78698 E+00$ & $-.11910 \mathrm{E}+02$ & DLAT 2 \\
\hline DLAT $\star$ DLON & $.15739 E+01$ & $-.43233 E+01$ & $.66245 E+01$ & DLAT $\star$ DLON \\
\hline DLON2 & $-.21617 \mathrm{E}+01$ & $.21229 E+01$ & $-.13381 E+01$ & DLON 2 \\
\hline DLAT 3 & $.42979 E+00$ & $-.24074 \mathrm{E} \quad 01$ & $-.14471 E+00$ & DLAT3 \\
\hline DLAT $2 \star$ DLON & $-.72222 \mathrm{E} \quad 01$ & $.32351 E+00$ & $.11547 \mathrm{E}+00$ & DLAT $2 \star$ DLON \\
\hline DLAT $\star$ DLON 2 & $.32351 E+00$ & $-.36370 \mathrm{E}+00$ & $.68370 E-01$ & DLAT $\star$ DLON 2 \\
\hline DLON 3 & $-.12124 E+00$ & $.16235 E+00$ & $-.15162 \mathrm{E}-01$ & DLON3 \\
\hline DLAT4 & $.62882 E-02$ & $-.10244 E-01$ & $-.36934 E-02$ & DLAT4 \\
\hline DLAT $3 \star D L O N$ & $-.40976 \mathrm{E}-01$ & $.61520 E-02$ & $.40920 \mathrm{E}-01$ & DLAT $3 \star$ DLON \\
\hline DLAT $2 \star$ DLON 2 & $.92280 \mathrm{E}-02$ & $-.55860 \mathrm{E}-02$ & $.79408 \mathrm{E}-01$ & DLAT $2 \star$ DLON 2 \\
\hline DLAT $\star$ DLON 3 & $-.37240 \mathrm{E}-02$ & $.23389 E-01$ & $-.62950 \mathrm{E}-01$ & DLAT $\star$ DLON 3 \\
\hline DLON 4 & $.58473 E-02$ & $-.62096 \mathrm{E}-02$ & $.27867 \mathrm{E}-0 \mathrm{I}$ & DLON 4 \\
\hline DLAT5 & $-.20117 \mathrm{E}-02$ & $-.70554 E-03$ & $-.38560 \mathrm{E}-03$ & DLAT5 \\
\hline DLAT $4 \star$ DLON & $-.35277 \mathrm{E}-02$ & $-.26323 E-02$ & $-.12395 E-03$ & DLAT 4 * DLON \\
\hline DLAT $3 \star$ DLON 2 & $-.52646 \mathrm{E}-02$ & $.78982 E-03$ & $-.86618 E-03$ & DLAT $3 \star$ DLON 2 \\
\hline DLAT $2 \star$ DLON 3 & $.78982 E-03$ & $.30752 \mathrm{E}-02$ & $.64792 E-03$ & DLAT $2 \star$ DLON 3 \\
\hline DLAT $\star$ DLON4 & $.15376 \mathrm{E}-02$ & $-.16880 \mathrm{E}-02$ & $-.53596 \mathrm{E}-03$ & DLAT $\star$ DLON 4 \\
\hline DLON 5 & $-.33760 E-03$ & $-.48307 E-03$ & $-.60981 E-03$ & DLON 5 \\
\hline DLAT6 & $--.75790 \mathrm{E}-04$ & $-.21558 \mathrm{E}-04$ & $.60118 E-04$ & DLAT6 \\
\hline DLAT $5 \star$ DLON & $-.12935 \mathrm{E}-03$ & $-.13645 E-03$ & $-.15450 \mathrm{E}-03$ & DLAT $5 \star$ DLON \\
\hline DLAT $4 \star$ DLON 2 & $-.34113 E-03$ & $.17082 E-03$ & $-.31680 \mathrm{E}-03$ & DLAT $4 \star$ DLON 2 \\
\hline DLAT $3 \star$ DLON 3 & $.22776 \mathrm{E}-03$ & $.19601 \mathrm{E}-03$ & $.53890 \mathrm{E}-04$ & DLAT $3 \star$ DLON 3 \\
\hline DLAT $2 \star$ DLON 4 & $.14701 E-03$ & $-.31929 E-03$ & $-.29349 \mathrm{E}-03$ & DLAT $2 \star$ DLON 4 \\
\hline DLAT $\star$ DLON 5 & $-.12772 \mathrm{E}-03$ & $-.38714 \mathrm{E}-05$ & $.26569 E-03$ & DLAT $\star$ DLON 5 \\
\hline DLON6 & $-.64510 \mathrm{E}-06$ & $.40350 \mathrm{E}-04$ & $-.65675 \mathrm{E}-04$ & DLON6 \\
\hline
\end{tabular}


netic charts showed smooth variation of geomagnetic field both on land and on adjacent sea. Figures 2 to 5 illustrate the spatial features for $D, H, Z$ and $F$ respectively. For the purpose of comparison similarly drawn isomagnetic maps using spherical harmonic coefficient corresponding to IGRF model of 1965.0 are superimposed in figures by broken line. The RMS values pertaining to deviation of observed data with respect to IGRF are also computed and included in table 3 . Ir may be noted that for purpose of computing RMS values, the deviations at dummy stations provided over the oceans are not considered.

\subsection{Analytical representation of temporal variation}

The behaviour of secular variation at Indian magnetic observatories has been studied by several workers (e.g. Bhargava and Yacob 1969: Yacob and Rangarajan 1973;

Table 3. Root mean square deviation of observed data with respect to polynomial as well as from IGRF

\begin{tabular}{ccc}
\hline & \multicolumn{2}{c}{ RMS Values (nT) } \\
\cline { 2 - 3 } Element & Polynomial & IGRF \\
\hline$X$ & 178 & 214 \\
$Y$ & 172 & 213 \\
$Z$ & 231 & 275 \\
$H$ & 178 & 214 \\
$F$ & 193 & 235 \\
$D$ & $0.25^{\circ}$ & $0.31^{\circ}$ \\
\hline
\end{tabular}

DECLINATION

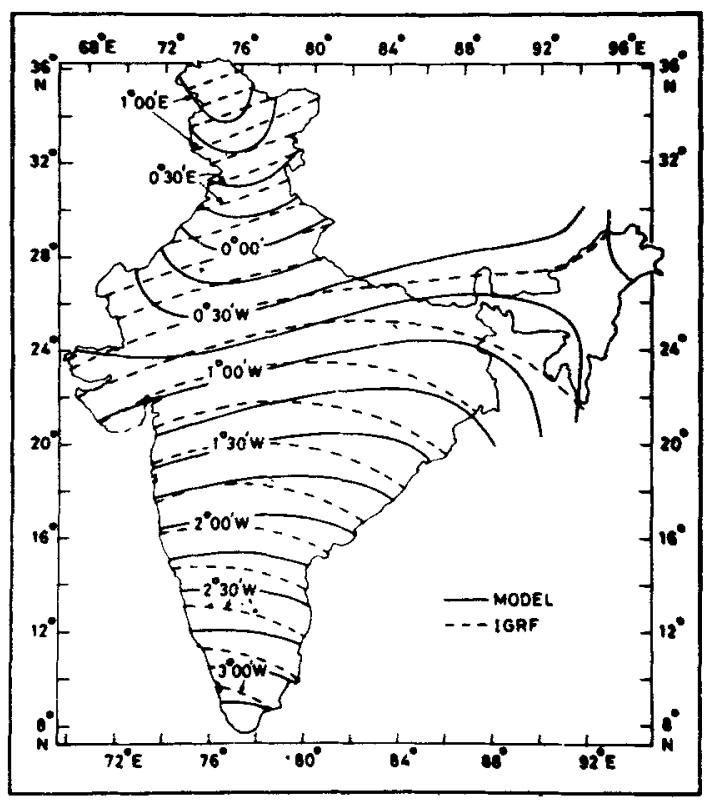

Figure 2. Isomagnetic charts of declination for the epoch 1965.0 obtained through polynomial model and IGRF. 


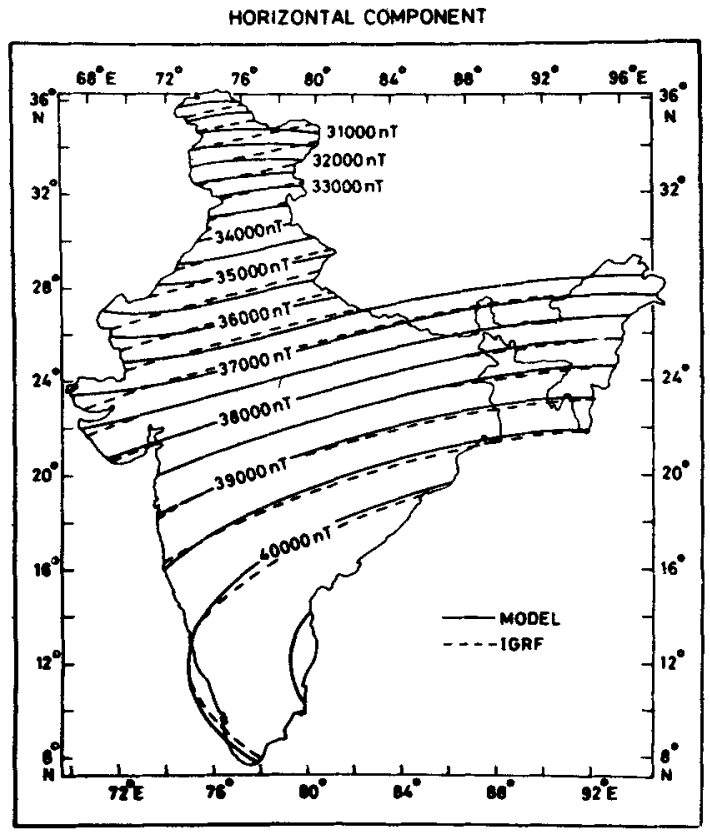

Figure 3. Isomagnetic charts of horizontal component for the epoch 1965.0 obtained through polynomial model and IGRF.

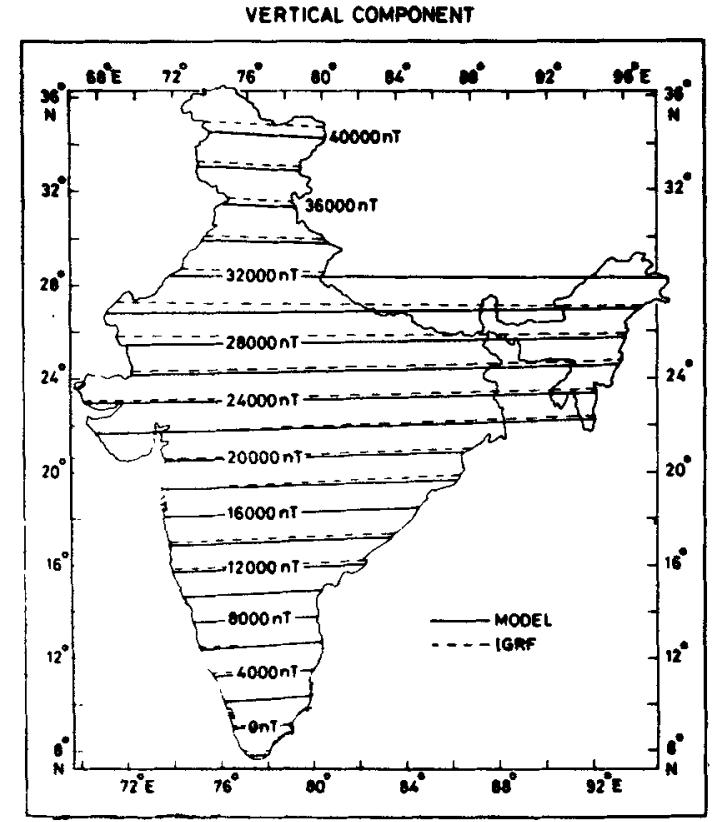

Figure 4. Isomagnetic charts of vertical component for the epoch 1965.0 obtained through polynomial model and IGRF. 


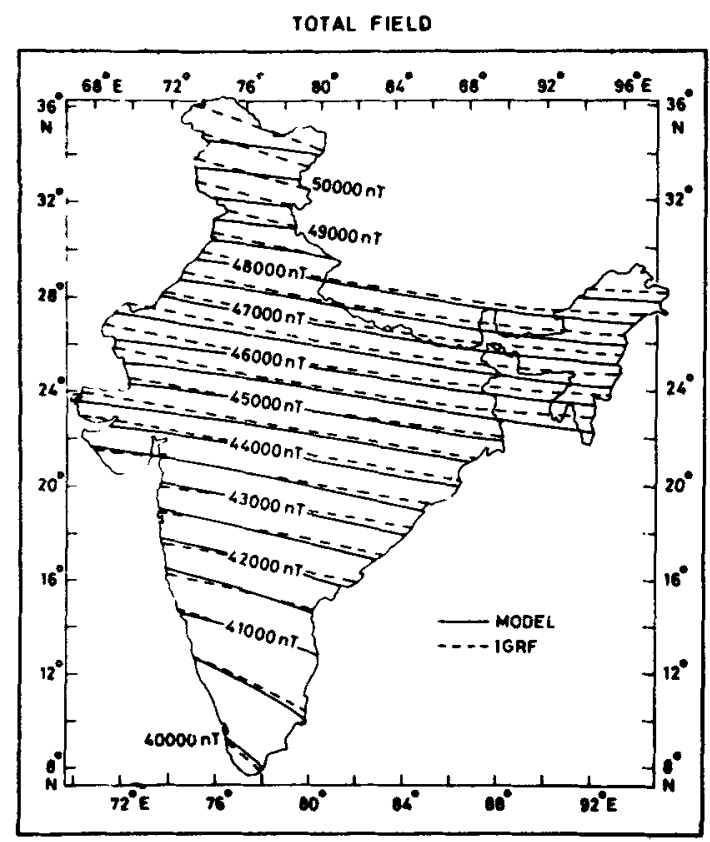

Figure 5. Isomagnetic charts of total force for the epoch 1965.0 obtained through polynomial model and IGRF.

Srivastava and Abbas 1977). Broad agreement in secular trends with some differences in detail is registered at various observatories. In order to bring out the regional and local characteristics of temporal variations over Indian region, a set of 93 uniformly distributed repeat stations, where observations in $H, D$ and $Z$ from all three surveys were available, were selected. From these observations, the field values corresponding to the epoch of the 1965.0 , i.e. input data used in modelling spatial variation, are subtracted. It may be emphasized that in the process of subtraction the constant part of the core field as well as magnetic field of crustal origin is effectively eliminated, leaving behind the component of the field which has varied in time with respect to 1965.0. With the aid of these residual data, the behaviour of secular variation as well as its spatial dependence on latitude and longitude were determined for any element by fitting a polynomial of the following form:

$$
\Delta C=n_{1} \Delta t+n_{2} \Delta t^{2}+\Delta \theta\left(n_{3} \Delta t+n_{4} \Delta t^{2}\right)+\Delta \lambda\left(n_{5} \Delta t+n_{6} \Delta t^{2}\right)
$$

$n$ 's are the coefficients to be evaluated and other notations are the same as defined in (1). This polynomial form is analogous to that adopted by Nevanlinna (1979) to model secular variation over Finland. It should be noted that by virtue of the nature of the defining residual field $(\Delta c)$ it tends to be zero when $\Delta t=0$, i.e. residual field is zero everywhere when $t=1965.0$. Using three observations from each of the 93 stations the $n$ 'coefficients were determined. An examination of the differences between observed values and those obtained through (8) shows that while, in general, the differences were small, there were a few observations which deviated appreciably from the 
computed values. Except for a few observations in the Koyna region, these anomalous observations are not consistent among elements and are scattered in time and locations which do not fit into the known tectonic framework of the country. The Koyna region of Deccan traps is known for its prolonged seismic activity beginning with 1966 or so. The detailed anomalous nature of geomagnetic anomalies is reported elsewhere (Arur et al 1980). Nevertheless, it should be noted that, in recent times, it has been reported from different parts of the world that in certain regions of very small areal extent secular variation at occasions tends to be anomalous. These anomalies are attributed to the seismo-magnetic effects arising due to the changes in the magnetization of rocks in the earth's crust under the accumulating mechanical stresses associated with earthquakes.

In order to minimise the effect of these sporadic observations on the polynomial fit, the observations with deviation of more than one standard deviation from computed values, which in no case exceed $7 \%$ of data, were excluded. From the remaining data, a fresh set of coefficients in (8) were re-evaluated. The coefficients in respect of elements $D, H, Z$ and $F$ are given in table 4 . As judged by the low RMS values, the fit appears fairly good. The accuracy of the coefficients is tested by comparing the trends of secular variation given by polynomial fit as against the observed variation at regular observatories. Figure 6 gives the secular variation in $D, H$ and $Z$ at the three widely separated magnetic observatories, namely Trivandrum (TRD), Alibag (ABG) and Sabhawala (SAB). The spatial dependence of secular variation as characterized by the secular change and secular acceleration, i.e. linear and quadratic parts of (8) are illustrated in figures 7 to 10 for $D, H, Z$ and $F$ respectively.

\section{Model fit}

The RMS deviations given in table 3 suggest that the field values computed through polynomials, worked out here, fit the observed data better than those represented by IGRF. This is in accord with the expected behaviour. The IGRF being evaluated through the spherical harmonic analysis of degree and order eight would account for only those magnetic features which have wavelengths greater than $5000 \mathrm{~km}$ whereas the polynomial of the field used here include contributions from anomalies as short as $1000 \mathrm{~km}$.

The isomagnetic charts given in figures $2-5$ show all the normal behaviour of geomagnetic field variations for such latitude range. The line of zero $Z$ variation, giving the location of dip equator, lay between Tirunelveli (Palayamkottai) and Ettayapuram, about $30 \mathrm{~km}$ to the north of Tirunelveli. This location differs from that given by Chatterji (1970) from the hand-contoured isomagnetic charts for the epoch 1965.0. He pointed out that dip equator passed through Tirunelveli. However, the present estimate is in good agreement with the position determined through ground magnetic survey undertaken jointly by IIG, NGRI and sol (1972) during 1971.

The spatial behaviour of geomagnetic field obtained through polynomial representation compares favourably with IGRF pattern, atleast in their primary features except in case of declination $(D)$. For this element the differences between IGRF and the polynomial model are appreciably large over the entire continent. In the case of $H, Z$ as well as total field, the field patterns given by the two models are more or less identical over the southern and central parts of the country but have a tendency to diverge progressively over the northern part. On the one hand it might be possible to 


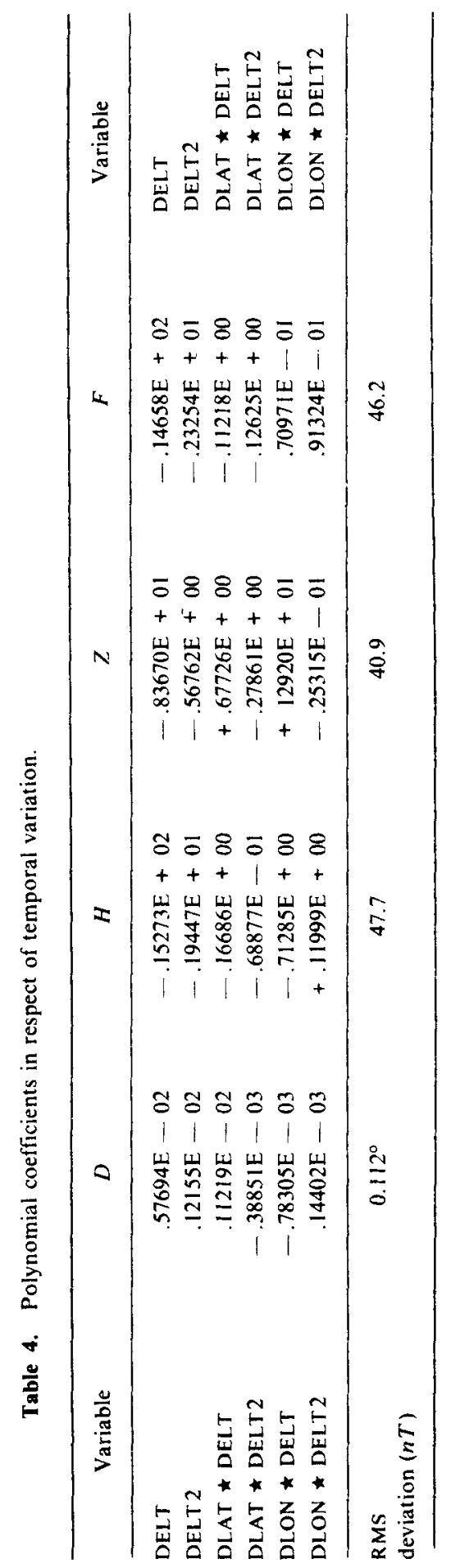


D
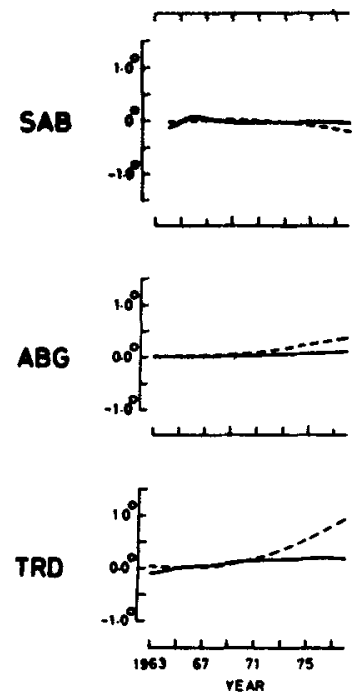

H
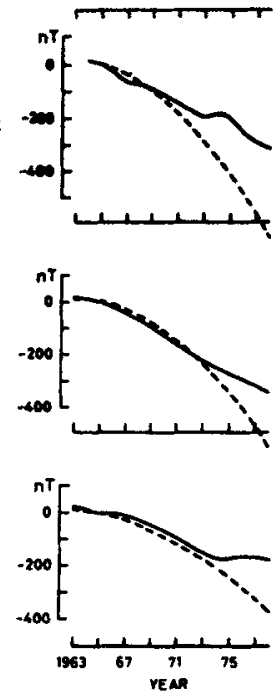

z
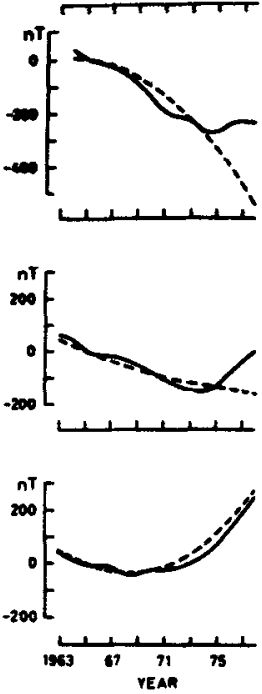

Figure 6. Comparison of observed and calculated (by polynomial fit) temporal variation in $D, H$ and $Z$ at Trivandrum, Alibag and Sabhawala.

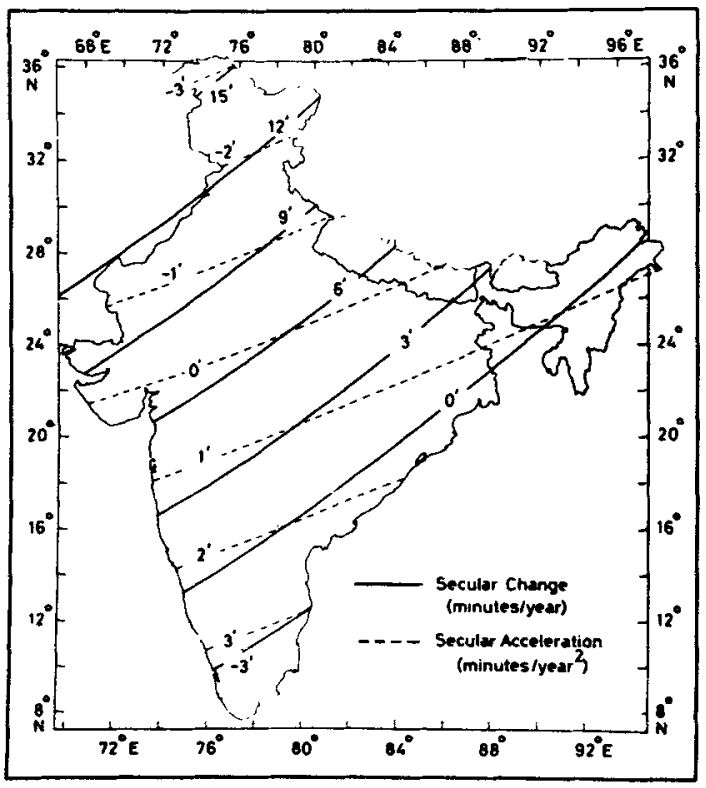

Figure 7. Spatial characteristics of secular variation in declination. 


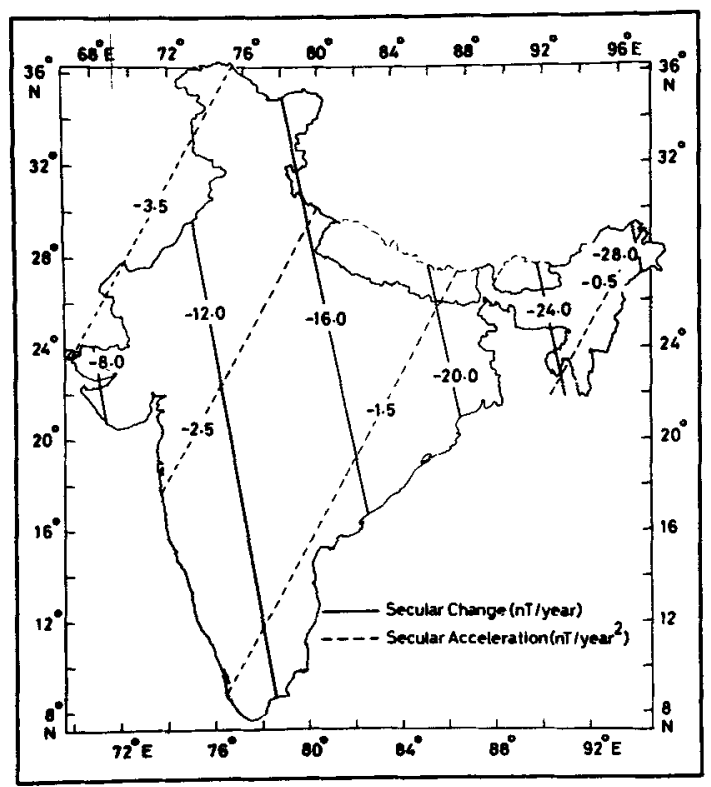

Figure 8. Spatial characteristics of secular variation in the horizontal component.

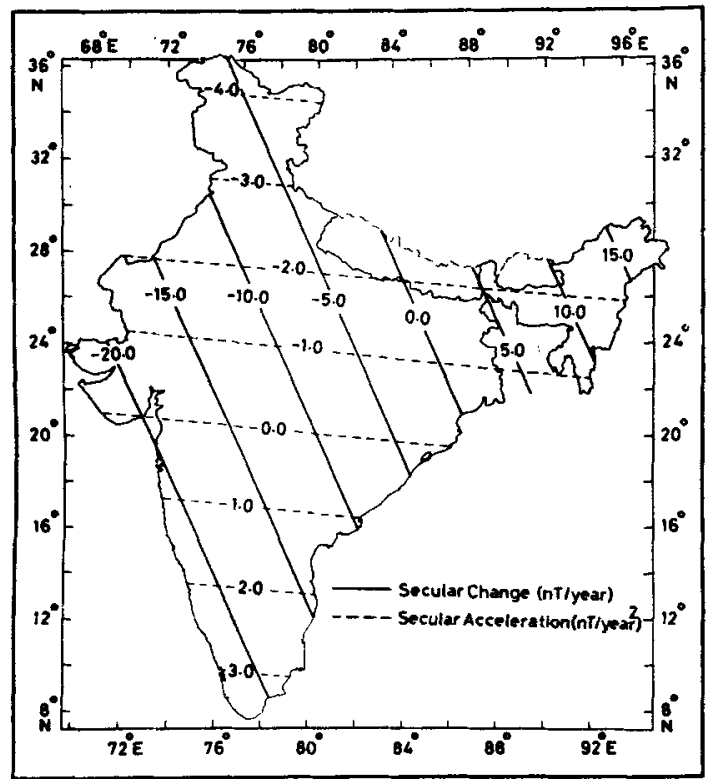

Figure 9. Spatial characteristics of secular variation in the vertical component. 


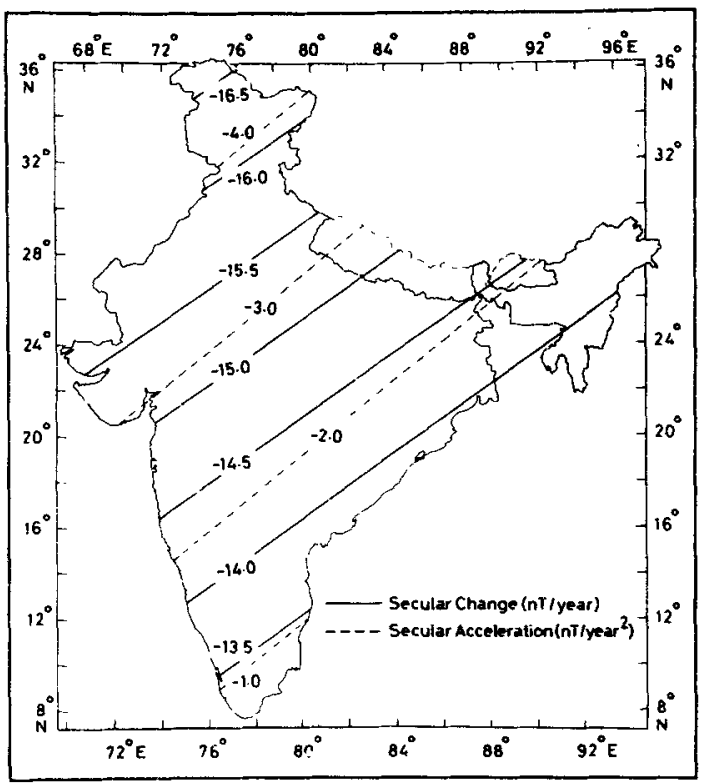

Figure i0. Spatial characteristics of secular variation in the total force.

attribute this divergence to the sparse distribution of magnetic measurements in the northern part of the country and on the other hand in view of the close proximity of this region to Himalayas, the differences between the two models could be indicative of the contribution from anomalies not included in IGRF model but incorporated in the present representation, i.e. anomalies with spatial scale lengths in the range of $1000-5000 \mathrm{~km}$. Such anomalies may arise from tectonic features in the deep crust or upper mantle under the Himalayas. It is interesting to note that magnetic anomaly maps compiled from pogo (Regan et al 1975) and MAGSAT (Langel et al 1982a, b) data clearly indicate a large negative anomaly over the Himalayas. In the present work the differences noticed between the present model and IGRF for $Z$ and $F$, elements most influenced by geological bodies, are also negative. Thus the divergence between the two models may be attributed to the influence of long wave anomalies over this region rather than to the paucity of the observations. Following this argument the similarity between the present model values and those of IGRF over the central and southern parts of the country would then imply the absence of deep structures in these parts of the country which could give rise to magnetic anomalies with wavelengths in the range of $1000-5000 \mathrm{~km}$.

As seen from figure 6, the behaviour of secular variation (sv) obtained by polynomial fit, at the three magnetic observatories, TRD, ABG and SAB, representing southern. central and northern parts respectively of the country, is reasonably close to that actually registered at these observatories, particularly during the period over which data have been acquired. SV trends for these stations evaluated using IGRF secular change coefficients corresponding to 1965 and 1970 fail to portray the true secular change even over the time-span of the order of five years for which the coefficients are. 
considered to be valid. While this clearly establishes the superiority of the polynomial method in the analytical representation of $s v$, it should be noted that outside the period of observations the differences between the observed and calculated values tend to increase, suggesting that polynomial coefficients have little predictive value. However, it might be added that certain short term changes registered in magnetic field around 1973-76, for example seen in $H$ traces of TRD and SAB in figure 6, might correspond to secular variation impulse (sudden change in the rate of secular variation) of the type reported by Alldredge 1975; Mizuno 1980; Nevanlinna and Sucksdorff 1981 . Obviously such impulses cannot be modelled by low degree polynomial as such polynomials are expected to reveal only the long-term trend in secularvariation. The most salient feature of the nature of secular variation to emerge from the inspection of time coefficients as well as charts of $s v$ given in figures 7-10 can be summarised as follows for different elements:

$s V$. in $D$ : At the southern tip of India, $\mathrm{sv}$ is dominantly determined by secular acceleration (SA) term and the resulting SV has easterly trend with respect to 1965.0 value. On the upper half of the country secular change (SC) and sa terms have opposite sign. Their relative strengths are such that $S A$ contribution would exceed the SC contribution around 1970, resulting in the reversal of sv trend.

$s v$ in $H$ : In the case of $H, \mathrm{sv}$ is dominantly linear. Except on the eastern flanks, sA and sc are additive in nature. There is a marked east-west asymmetry in the rate of secular variation per year, the decrease being more pronounced on the eastern part of the country than on the western part.

$s v$ in $Z$ : Nature of $\mathbf{s v}$ in $Z$ tends to be much more complex with a strong spatial dependence. In the northern part, $S A$ and SC are negative indicating that vertical component is decreasing with increasing rate year after year. In the southern part of the country SC and SV have a tendency to arrest each other. The SC controlling the SV during the years 1963-68, thereafter SA determines the behaviour of SV, the reversal in SV occurring around the year 1968-69.

sV in $F$ : SC as well as SA contributions have negative trend all over the country, suggesting a continuous decrease in the total field of earth over this part of the earth. Isopor run NE-SW, with their magnitude increasing with increasing latitude.

\section{Conclusion}

The present study clearly shows that polynomial representation, adopted here, satisfactorily depicts various features of smoothly varying geomagnetic field consistent with the general behaviour of the geomagnetic field for these latitudes. Comparison with IGRF discloses certain long wavelengths anomalies in the northern part of the country. The polynomials giving spatial and temporal behaviour of geomagnetic field can be conveniently combined to evaluate analytically the magnetic field values at any location in the country for any instant of time in the period interval of 1963-73. Polynomial expressing temporal changes proves less reliable if extended beyond the period of observations. The life period of such modelled representation could be regularly updated by adding data from future surveys and recomputing the coefficients of (8). There might also be scope to improve the accuracy of spatial polynomial with the addition of data from surveys over the seas. 


\section{References}

Alldredge L R $1975 \mathrm{~J}$. Geophys. Res. 801971

Arur M G, Bains P S and Jeevan Lal 1980 Geophys. Res. Bull. 1867

Bhargava B N and Yacob A $1969 \mathrm{~J}$. Geomagn. Geoelectr. 21385

Blakely R J and Cande S C 1979 Rev. Geophys. Space Phys. 17204

Bullard E C 1967 Earth Planet. Sci. Lett. 2293

Chapman S 1942 Terr. Magn. Atmos. Elec. 471

Chatterji J 1970 Proc. Sem Probls. Equat. Electrojet Aug 1970 Ahmedabad (PRL) Paper 8

Dawson E and Newitt L R 1977 Can. J. Earth Sci. 14477

Fabino E B and Peddie N W 1969 ESSA Tech. C \& GS 38

Hinze W J 1979 Rev. Geophys. Space Phys. 17267

IIG, NGRI, SOI 1972 Geophys. Res. Bull. 10167

Langel R A. Phillips J D and Horner R J 1982a Geophys. Res. Lett. 9269

Langel R A. Schhetzler C C, Phillips J D and Horner R J 1982b Geophys. Res. Lett. 9273

Mizuno H $1980 \mathrm{~J}$. Geomagn. Geoelectr. 32413

Nagata T 1971 in World magnetic survey 1957-1969 (ed.) A J Zamuda IAGA Bull. No. 28, 36

Nevantina H 1979 J. Geophys. 46201

Nevantinm $H$ and Sucksdorf 1981 J. Geophys 5068

Regan R D. Cain J C and Davis W M 1975 J. Geophys. Res. 80794

Srivastava B J and Abbas H 1977 J. Geomagn. Geoelectr. 2951

Yacob A and Rangarajan G K 1973 Pure Appl. Geophys. 112286 